\title{
English and Arabic Inscriptions in the Linguistic Landscape of Yemen: A Multilingual Writing Approach
}

\author{
Anwar A. H. Al-Athwary \\ Department of English, Faculty of Arts and Science, Najran University, Saudi Arabia \\ E-mail: anwaraha@yahoo.com
}

Received: 09-12-2016

Published: 01-07-2017
Accepted: $23-02-2017$

doi:10.7575/aiac.ijalel.v.6n.4p.149
Advance Access Published: April 2017

URL: http://dx.doi.org/10.7575/aiac.ijalel.v.6n.4p.149

\begin{abstract}
The present paper investigates the multilingual written texts of the signboards in the public space of Yemen. It attempts to apply Reh's (2004) typology of multilingual writing. Reh introduces four strategies of multilingualism: duplicating, fragmentary, overlapping, and complementary. They refer to the arrangement of information in the inscriptions of multilingual signs in a given linguistic landscape (LL). To achieve this purpose, a data corpus of 755 multilingual signs in the LL of Yemen has been used, the majority of which are bilingual in Arabic and English. The analysis showed that all four strategies of duplicating, fragmentary, overlapping, and complementary multilingual writings were generally employed in Sana'a's LL. While overlapping and complementary multilingualism were totally absent in the top-down signs, duplicating and fragmentary multilingualism had much higher frequency over overlapping and complementary ones in bottom-up signs. Keeping in mind that speech community in Yemen is monolingual in Arabic, the absence or low frequency of overlapping, and complementary signs in both top-down and bottom-up levels can be explained by the fact that these two types of texts presuppose multilingual readers since knowledge of all the languages involved is necessary to understand the whole message. The model of writing mimicry system proposed by Sutherland (2015) is also examined. Writing mimicry system was found to be a salient feature of the public space of Yemen performing some specific functions; it is only used for advertising and promotional purposes rather than expressing the identity of ethnolinguistic minorities. The study also revealed that Sana'a multilingual LL is characterized by the use of Arabicised English, glocalisation and multifunctional signs, all of which are employed to serve a general purpose of promoting, and advertising commodities and showing modernity and success. Standard Arabic appears on almost all of both top-down and bottom-up signs. The scarce use of Yemeni Arabic is indicative of the notion of Arab nationalism. Linguistic nationalism refers to the communicative and symbolic functions of Standard Arabic in articulating national identity in the LL of Yemen.
\end{abstract}

Keywords: Linguistic landscape, duplicating, fragmentary, overlapping and complementary multilingual writing, writing system mimicry

\section{Introduction}

\subsection{Linguistic landscape}

The relationship between language and landscape (public space) is inevitable and each one requires the other. Nash (2016: 380) depicts this relationship by arguing that "language and landscape are obliged to each other. Language demands landscape, landscape expects language." Linguistic landscape (LL) is a relatively new area of sociolinguistics that focuses on written languages in the public space of a given territory. Landry and Bourhis (1997) represents a turning point in the LL studies after which the LL has gained importance in the field of sociolinguistics. They view the LL as a newly established approach in the field of language policy and planning, which aims to examine multilingualism in speech communities.

The unit of analysis in a given LL is a sign. Signs function as illustrative texts which can be read, photographed, probed and linguistically and culturally dissected. According to Backhaus (2007), signs are used in order to disseminate messages of general public interest such as topographic information, directions, warnings, etc. Public signs also appear in commercial contexts like marketing and advertising, where their function is to draw attention to a business or product. Apart from the messages that these public signs intend to convey as mentioned earlier, we can also learn the diversity of language and culture underlying in the messages because public signs are "a type of semiotic sign in that they stand for something other than themselves" (Akindele, 2011: 2).

Despite the fact that speech community in Yemen is generally monolingual in Arabic, the public space of Yemen is primarily multilingual. Al-Athwary (2012) reports that the majority of the stationary written texts in the streets of Sana'a are bilingual in Arabic and English. Arabic is the native and official language in Yemen where Standard Arabic and other Arabic dialects are used in what is known as the diglossic situation. In addition to Arabic, Mehri and Socotri are two ancient non-Arabic dialects which are used as the first language by a small group of citizens in very restricted areas 
(Al-Athwary, 2012). They are purely spoken languages which are neither used in the official interactions nor in the public space of Yemen. Recently, Yemen has been influenced by the processes of modernization and globalization through mainly the medium of English. English has the status of a foreign language in the country. It has been given a special attention and employed in many spheres of Yemeni state of affairs like education, mass media, the internet and commercial and business activities and (Al-Athwary, 2014).

Multilingual writing on signs has increasingly become a salient feature of the LL around the world. Cenoz and Gorter (2008: 270) emphasize that "the idea of monolingualism by country - one state, one language- has become obsolete and has been overtaken by a complicated interplay of many languages" and "monolingual countries were always an exception, but globalization with its ensuring migration flows, spread of cultural products, and high spread communication has led to more multilingualism. In current approaches to multilingual writing, written languages are the focus of the LL studies."

The next subsection will shed some light on Reh's (2004) typology of multilingual writing and Sutherland's (2015) insights on writing system mimicry as a new phenomenon that has emerged in the LL studies.

\subsection{The theoretical background of the study}

\subsubsection{Multilingual writing approach}

Reh (2004) develops a model for the study and categorization of multilingual writings as encountered in the streets. This model involves three parameters of multilingual writing: a) spatial mobility of signs, b) visibility of multilingualism, and c) information arrangement on signs. Of the three parameters that she proposes, the third is the most interesting in the context of the present study as it is helpful in the analysis of multilingual signs. This parameter relates to the way in which information is arranged in a multilingual text. Four main types of arrangement are distinguished: a) duplicating, b) fragmentary, c) overlapping and d) complementary.

Duplicating multilingual writing relates to those written texts in which the same amount of information is given in more than one language. This practice reflects the presence of what Reh (2004: 8) calls "societal multilingualism", a term referring to "the existence of more than one language in the target community". This type of multilingualism may arise out of technical and affective aspects of communication. The former occurs in situations where "individual multilingualism" is insufficient in a certain multilingual setting in the sense that not all people in the target community will be reached by a single language only. It is also relevant in situations in which the multilingual writer is mainly interested in reaching a particular target group, particularly in the cases of trade and tourism. The latter form of duplicating multilingualism comes into existence when individual multilingualism is widely used to the extent that the LL texts are understood if inscribed in just one language.

Fragmentary multilingualism refers to multilingual inscriptions in which the full text is only presented in one language and certain parts of the same text are interpreted into another language or other languages.

The term overlapping multilingual writing is used if part of the information is given in two or more languages, and in all the languages also different pieces of additional information are given. In this case the content of the texts in the different languages may simply overlap, or the content may be identical although the speech acts are not. Even though Reh (2004) draws a distinction between fragmentary and overlapping multilingual texts, Backhaus (2007) considers both types exactly the same and term them as mixed signs.

Finally, complementary multilingualism occurs when texts in which different parts of the overall information are each rendered in a different language.

The last two types of multilingual text (overlapping and complementary) presuppose multilingual readers since knowledge of all the languages involved is necessary to understand the whole message. Reh found that in the case of English-Lwo inscriptions on buildings, signposts and posters in public places in Lira (Uganda), examples of complementary and overlapping multilingualism were much more frequent than those of duplicating or fragmentary multilingualism. According to Reh (2004: 38), the analysis of multilingual text types and their communicative functions enables conclusions to be drawn "regarding, among other factors, the social layering of the community, the relative status of the various societal segments, and the dominant cultural ideals of the community". In terms of translation, duplicating multilingualism corresponds to word-for-word and free translation. Partial translation may either be fragmentary or overlapping, and no translation means that the information given is complementary.

Next to this, Reh (2004) distinguishes between visible multilingualism and covert multilingualism. Visible multilingualism means that you can see the different codes without moving a foot. In most instances of visible multilingualism, the different codes are on the same physical sign.

The taxonomy of combinations of languages and information in the text on signs proposed by Reh (2004) was useful for the later LL studies (e.g., Alomoush, 2015; Backhaus, 2007; Edelman, 2010; Wielfaet, 2009).

\subsubsection{Writing system mimicry}

The term writing system mimicry (WSM) is first introduced by Sutherland (2015) to fill a gap related to written languages uses in the public sphere. WSM is described as the choice to make a text in one writing system superficially resemble text in another within the LL. Although WSM is a distinctive feature of the LL that is a rich source of sociolinguistic research, it is not restricted to this domain. Sutherland (2015) defines WSM as follows: 
The mapping of (real or imagined) design features and/or graphemes of a mimicked writing system onto a base writing system, so that the base writing system somewhat resembles the mimicked writing system while retaining legibility. (Sutherland, 2015: 150)

The 'base writing system' here is the lens through which the text is intended to be read. The choice to mimic another writing system may be conducted to serve some social or commercial purposes that can be interpreted sociolinguistically; WSM is employed purposefully, either for advertising or identity purposes, or both, and the identity can be ethnic or social. Sutherland reviews all academic as well as folk terms which have been used in the literature to refer to the process of mimicry of written texts. He argues that with the exception of 'typographic mimicry' introduced by Coulmas (2014), all terms like Faux x, Foreign look font, Mimicry typefaces, etc. refer to the product of the phenomenon rather than the phenomenon itself, implying that there is some kind of standard process for creation and more-or-less uniform degrees of implementation. Sutherland avoids using the term 'typographic mimicry' coined by Coulmas (2014), because this term, as he argues, refers to "the act of arranging type, and is therefore too broad for current purposes, as it could reference any design elements of typography".

WSM has recently become an apparent feature of the LL, especially on signs of nongovernment service sectors and private commercial enterprises. It is only employed on bottom-up signs rather than on top-down ones to have the functions of identity and solidarity. In addition to the functions of identity and solidarity that WSM may serve in the LL, it also works as an instrumental device since it is a very productive tool in a situation where more than one language is used.

Sutherland concludes that the research questions that he has addressed are still too broad and need further investigations; there is still a lack of uniform terminology; the new phenomenon of WSM should be investigated not only as an expression of identity and a marketing tool but also as a phenomenon that may perform the functions of both instrumentality and symbolism.

\subsubsection{Top-down and bottom-up signs}

Ben-Rafael, Shohamy, Amara, and Trumper-Hecht (2006) examine signs in a 'top-down' and 'bottom-up' dichotomy. The former refers to the public and institutional signs and the latter refers to the private and individual ones. The distinction between top-down and bottom-up signs is another factor which contributes to the comprehension of language policy. Ben-Rafael (2009: 49) emphasizes that the distinction between top-down and bottom-up signs is significant because different signs are made by different actors for different audiences, and while top-down signs "serve official policies", bottom-up signs "are designed much more freely". Likewise, Backhaus (2007) uses these two terms in alternation with official and nonofficial signs.

The study of LL can also be interesting because it can provide information on the differences between the official language policy that can be reflected in top-down signs such as street names or names of official buildings and the impact of that policy on individuals as reflected in bottom-up signs such as shop names or street posters. LL is an instrument through which, language policy is reflected. In other words, the LL symbolizes the legitimacy and priority of certain languages over other languages. Spolsky (2004) states that the real language policy of a community is likely to be found in its practices than in the management of the policy. In support, Cenoz and Gorter (2006) affirm that there is the presence of an overt and covert policy in informing the practices in the linguistic landscape. While there are official policies that guide the setting up of street names and other signs, there is the impact that operates bottom-up from the signs and posters that form impressions on how the policy is negotiated. In this way, there could then be an unofficial policy that could have far-ranging influence on the linguistic landscape.

Top-down signs in Yemen are regulated by local authorities. The majority of top-down signs are prepared bilingually in Arabic and English according to municipal evidence taken from discussion between the author and some local authority's officials, "the scarce presence of governmental signs, especially street and area names, however, indicates the lack of language policy on the part of the government and the local authorities" (Al-Athwary 2012: 25).

Regarding the bottom-up signs, the local authorities have nothing to do with the issue of signs except in giving permits to put signs on the facades of stores. There is no policy to impose the use of English or any other language/s. It is the shop owners who decide whether to use English or not.

\section{Purpose of the study}

Sana'a's streets are condensed with written advertising information. Commercial enterprises, public and nonpublic service sectors have changed the LL in Yemen into an area of vast visual information, where both image- and languagebased information is combined together. Although Yemen is known as a typical monolingual society, the LL of Sana'a shows a growing degree of multilingualism. Moreover, English has a high prestige there and its increasing presence in the visual scenery of the streets of Sana'a has become part of everyday Yemeni life (Al-Athwary, 2012; Buckingham and Al-Athwary, 2016).

The present study is concerned with multilingual writing and thus investigates the inscribed signs in the public space of Yemen. It gives special attention to the amount of information in each language in multilingual signs and their intended readership. In other words, this study tries to examine language-spatial relationships and how the inscribed information of the displayed languages is arranged in the different types of signs of the LL of Yemen.

In order to achieve this purpose, Reh's (2004) model of multilingual writing typology is applied to the multilingual practices in the public space of Yemen. More specifically, the proposed parameters of information arrangement will be 
examined across the whole collected data. In doing so, the study will attempt to figure out to which extent English inscriptions are represented in the multilingual public space of Sana'a. Further, the study seeks insights from other works on multilingual writing approaches, mainly Sutherland (2015).

\section{Review of the literature}

The LL research has recently expanded to include not only street shop signs but also the indoor signage such as universities, railway stations, etc. (Haynes, 2012; Singhasiri, 2013; Wielfaert, 2009) as well as linguistic netscape (Troyer, 2012). Cenoz and Gorter (2008) go even further to claim that LL may have something to do with second language acquisition. They conclude that LL is a learning context and can also be used for raising awareness in second language acquisition. On the other hand, some works on the LL go deeper in the field to address very specific aspects of the LL. Tufi and Blackwood (2010), for instance, examine in detail and from a methodological perspective the brand names in the urban space seeking to identify a framework for a linguistic classification of brand names. Similarly, the focus of Anuarudin, Heng and Abdullah (2013) is only on one type of signs, that is billboards (large signboards on the streets/ roads usually used for advertising; hoardings). Anuarudin et al., (2013) discuss the linguistic practices, code choices and language policy of billboard advertisements in a selected cityscape. In this regard, Gorter (2013: 203) reports that "a promising direction in the LL studies consists of investigations of semipublic institutional contexts, such as government buildings, libraries, museums, hospitals, and schools. Education as an institution has already attracted the attention of some scholars."

An overview of the main developments in the field of LL studies indicates an increasing interest. Although LL is a very recent field of research, which was practically initiated by Landry and Bourhis (1997), a tremendous number of studies on LL have been comparatively conducted in many areas across the world. In his overview of the main developments in the field of LL studies, Gorter (2013: 190) confirms that "there is a large number of research projects and publications in applied linguistics in the use of written texts in urban spaces, especially in bilingual and multilingual settings". The thirty-two LL studies stated by Alomuosh (2015) have investigated many regions in Europe, Northern America, Africa and Asia, and represent only about one-fourth of the overall LL works so far. Regarding the use of English in the multilingual LL, Bolton (2012: 31) highlights that "the intrusion and use of English in the public spaces of the world's cities alongside national languages and local languages has received increasing attention over the last fifteen years or so".

On the other hand, issues that have been investigated so far in the field of LL vary a lot. Nash (2016: 130) reports that "sign research in the LL studies analyzes the relative visibility and salience of languages in the landscape, the authorship process, and policy implications, among other points of consideration". In other words, the LL research attracts our attention to topics such as LL symbolic and cultural functions (Al-Athwary, 2012; Ben-Rafael et al., 2006), language policy (Haynes, 2012), language minorities (Alomoush, 2015; Cenoz and Gorter, 2006), advertising (Buckingham, 2015 ; Buckingham and Al-Athwary, 2016; Tufi and Blackwood, 2010), the use of English in the public space (Al-Athwary 2014; Bolton, 2012) and methodological developments (Clemente, Vieira, Martins, and Andrade, 2013; Gorter, 2013; Puzey, 2016)

Ben-Rafael et al. (2006), for instance, emphasize on the symbolic function of LL in shaping the public space of some cities in Israel (occupied Palestine) and in occupied East Jerusalem. Further, they make a clear distinction between "topdown" and "bottom-up" signs. Such signage process is affected by behaviors and choices of what the authors call "LLactors", that is the people who concretely participate in the LL make up. Both types of signs which are designed by LLactors contribute to the symbolic construction of the public space. Al-Athwary (2012) likewise emphasizes that in addition to the informational and symbolic functions, LL seems to have a peculiar function of being used as a cultural and folkloric marker by inscribing mnemonic devices on LL objects that hark back to the earlier heritage and culture of a given LL.

Puzey (2016), which is the most recent work on LL, provides a detailed account on major theoretical and methodological developments in the LL research, and in the closely related areas of geosemiotics and semiotic landscapes. The study also outlines the onomastic dimension of LL approach, including opportunities to apply the LL fieldwork techniques to study observable onomastic practice, to facilitate the collection of names, and to study names in relation to language policy, as well as the benefits of LL approach for the study of commercial and corporate names, and the commodification of names.

In reviewing the literature on the field of LL, much sociolinguistic analysis has been attributed to the importance of English in the LL studies. Even though English competence is not high among Yemeni people, English widely appears on LL signs in the LL of Sana'a. With regard to private spaces, English is competing Arabic on LL items. In this part of the world, English is regarded as 'the language of upward social mobility, as new, prestigious and desirable to learn'. Al-Athwary (2014), for example, focuses on shop signs which display translation errors or deviant forms of English in Sana'a's LL. Most deviant expressions were found to occur at three main linguistic levels: orthographic, morphosyntactic and lexical levels.

As far as multilingual writing practices in the public space are concerned, several works have been contributed to this area. In addition to Reh (2004), Sebba (2012), and Sutherland (2015) whose theoretical principles have been discussed above in order to be applied in the present study, the LL studies like Coulmas (2014), and Seargeant (2013) are also important contributions. The common main concern of the two studies is to probe into the factors which stand behind the choice of writing system/ script either in language contact situation in its written mode (Coulmas, 2014) or in the 
semiotic work performed by signs in an LL context (Seargeant, 2013). Both emphasize that social and cultural consideration play a great role in script choice.

Although Arab countries like Iraq, Palestine, Algeria, and Morocco represent, in particular, areas of linguistic diversification and language minorities and deserve research from various aspects of LL (Al-Athwary, 2012), little attention has been paid to LL of the Arab countries in comparison to other parts of the world. In the last decade, however, a number of the LL studies in the Arab countries have emerged. Among these are Al-Athwary (2012), and AlAthwary (2014) in Yemen, Alomoush (2015) and Nofal and Mansour (2014) in Jordon, Buckingham (2015) in Oman, Buckingham and Al-athwary in Yemen and Oman (2016), and Ben Said (2010) in Tunisia. All of these studies tackle various themes related to LL like those mentioned above, those of language visibility and dominance, language policy and minority, the use of English and so on. None of them addresses the issue of multilingual writing practices in the public signage except Alomoush (2015). Alomoush adopts, though briefly, Reh's (2004) typological model of multilingualism in analyzing how written texts are combined in the public space of Jordon. The study generally shows that the overwhelming majority of sings contain complementary multilingual writing while fragmentary multilingualism represents the lowest number of signs. No numerical data have been given to show the different tendencies between topdown and bottom-up toward the four multilingual writing strategies defined by Reh (2004). All this will be critically compared to the results obtained from analyzing the collected data of the present study.

The public space in Yemen has been examined from the point view of LL functions (Al-Athwary, 2012), deviant forms of English in Sana'a's LL (Al-Athwary, 2014), and the use of English as an advertising tool in Sana'a's LL as compared to that of Muscat in Oman (Buckingham and Al-Athwary, 2016). The investigation of written texts in the Yemeni LL from a multilingual approach has not been addressed yet. Taking this gap in the literature into consideration, the current study seeks to find answers to the following research questions regarding multilingual writings practices in the public space of Yemen:

1. How are written multilingual inscriptions arranged in the signs of Sana'a's Streets? Or to which extent Reh's (2004) four multilingual writing strategies of complementary, duplicating, fragmentary, and overlapping multilingualism are applied in the LL of Yemen?

2. Do top-down signs show the same tendencies toward the four multilingual writing strategies like bottom-up signs?

3. What role does writing system mimicry (if any) play in the LL of Yemen? Or Which features are mimicked in the LL of Yemen and why?

\section{Research methods}

The present study locates in sociolinguistics, more specifically in the area of linguistic landscape. In order to answer the research questions stated in the literature review above, a qualitative method is used to describe and analyze the English and Arabic written texts appearing on the bilingual signs in Sana'a LL. The data corpus of such signs is primarily employed numerically to account for the frequency of occurrence of the four strategies of multilingual typology proposed by Reh (2004): duplicating, fragmentary, overlapping, and complementary. Then, each strategy is analyzed with appropriate illustrations from the corpus. The study concludes with a discussion of the most significant findings that have been obtained in the analysis.

In order to be studied, classified and analyzed, signs should be primarily photographed. The practice of LL studies in sign collection is to photograph the signs by using a digital camera, code them for significant features, and make statistical comparisons to draw conclusions about the different written languages displayed in that area (see Ben-Rafael et al., 2006; Edelman, 2010; Gorter, 2013; Sutherland, 2015).

The data used for this study is a total of 755 multilingual signs, almost all of them are bilingual in Arabic and English. They include both top-down (59) and bottom-up (696) signs. They are a part of a larger data corpus (comprising 1500 signs) photographed in four streets in the capital city of Sana'a in 2012 by a digital camera. All written data in English and Arabic appeared on the multilingual signs were inserted on Microsoft Excel worksheets. The signs were primarily analyzed and sorted out according to multilingual writing taxonomy introduced by Reh (2004): duplicating, complementary, fragmentary and overlapping. Further, the analysis of excel files reveals others points to be considered such as writing system mimicry, multifunctional signs, Romanized Arabic (RA) vs. Arabicized English (AE), and glocalisation.

The public space of Yemen is heavily decorated by both monolingual and multiannual signboards with different proportions (see Al-Athwary, 2012). Monolingual signs are excluded. As mentioned above, Reh (2014) distinguishes between visible multilingualism and covert multilingualism. The latter type represents a very interesting area of multilingualism. The collected data, however, are void of covert multilingual signs. This study, therefore, focuses on visible multilingual signs which are mainly Arabic-English bilingual signs. Although the overwhelming majority of signs are bilingual in Arabic and English, the cover term "multilingual" is used instead. When talking about multilingual writing strategies, terms like complementary bilingualism, etc., might be used instead of complementary multilingualism, etc.

This study investigates only signboards which are relatively stationary (physically fixed) rather than movable ones. This is because the stationary signs are more durable and consequently more expressive in conveying the symbolic message of a given public space than the non-permanent ones (Al-Athwary, 2012). Moreover, "inscriptions on stationary objects 
presuppose a spatially mobile readership, since the text and its potential readership would otherwise not coincide" (Reh, 2004: 3). These include both public/ institutional signs (street names, hospital names, traffic signs, etc.) and also private/ individual signs (commercial shop signs, etc.). In the literature on LL, the former is referred to as "top-down" signs and the latter as "bottom-up" signs (see Ben-Rafael et al., 2006). Both official and non-official Bilingual/ multilingual signs are to be included in the analysis. In this study, the researcher has adopted 'top-down' and 'bottomup' terms.

\section{Analysis and results}

The 755 multilingual signs, both top-down and bottom-up, have been analyzed from a multiannual writing approach in order to know how information in such signs is arranged. The numerical data presented in Table 1 reveal that Reh's (2004) four strategies of duplicating, fragmentary, overlapping and complementary multilingualism are at work in the public space of Sana'a with varying degrees of frequency. The use of complementary multilingualism (24 signs with $03.18 \%$ ) in Sana'a's LL is really striking and beyond the expectations (reasons behind the unexpected use of this strategy will be elaborated when talking about the frequency of the four strategies in bottom-up sign in Table 2 below). The analysis also shows that the overwhelming majority of signs contains duplicating multilingual writing while overlapping multilingualism represents the lowest percentage.

Table 1. Distribution of multilingual strategies in the whole corpus

\begin{tabular}{lll}
\hline Strategies of multilingual writing & No. & Percentage \\
\hline Duplicating multilingualism & 362 & 47.95 \\
\hline Fragmentary multilingualism & 352 & 46.62 \\
\hline Overlapping multilingualism & 17 & 02.25 \\
\hline Complementary multilingualism & 24 & 03.18 \\
\hline Total & 755 & 100 \\
\hline
\end{tabular}

The above results are not in line with those of Reh (2004). In the case of Arabic- English inscriptions in the streets of Sana'a, the examples of duplicating and fragmentary multilingualism are much more frequent than those of overlapping or complementary multilingualism. The opposite is true in the case of English-Lwo in the town of Lira in Uganda. Reh finds that instances of complementary and overlapping multilingualism were much more frequent than those of duplicating or fragmentary multilingualism. This is simply because the speech community in Uganda is multilingual in English, Lwo and one more local language while it is monolingual in Arabic in Yemen. Readers in Lira town find no difficulty in interpreting complementary and overlapping signs because most of them are bilingual in more than one language, but people in Sana'a's streets cannot do so.

Then, the corpus is analyzed by the sign type, i.e. top-down and bottom-up signs. Table 2 shows that while the four strategies are involved in the inscriptions of bottom-up signs, only the two strategies of duplicating, and fragmentary are presented in top-down signs but overlapping and complementary multilingualisms aren't. The other difference between top-down and bottom-up signs lies in the fact that duplicating multilingual writing represents the highest frequency among the former whereas it is not the case with the latter. This occurs because of the local municipality language policy where the majority of top-down signs are prepared bilingually in Arabic and English and in the same time information in Arabic are rendered into English.

Table 2. Distribution of multilingual strategies by sign category

\begin{tabular}{lllll}
\hline \multirow{2}{*}{ Strategy } & \multicolumn{2}{l}{ Top-down signs } & \multicolumn{2}{l}{ Bottom- up signs } \\
\cline { 2 - 5 } & No. & $\%$ & No. & $\%$ \\
\hline Duplicating & 45 & 76.27 & 317 & 45.55 \\
\hline Fragmentary & 14 & 23.73 & 338 & 48.56 \\
\hline Overlapping & --- & --- & 17 & 02.44 \\
\hline Complementary & --- & --- & 24 & 03.45 \\
\hline Total & 59 & 100 & 696 & 100 \\
\hline
\end{tabular}

The fact that signs in complementary multilingual writing, as articulated by Reh (2004), are the overwhelming majority of bottom-up signage reflects the involvement of other language varieties widely used by the local community. This view holds true in the LL of Jordan where complementary multilingualism is more dominant than other types of multilingual writing (see Alomoush 2015: 276) because of the use of Jordanian Arabic and European languages in addition to standard Arabic. The case in Yemen, however, is different. The low frequency of complementary 
multilingualism in Sana'a's LL can be explained by the fact that Yemeni colloquial Arabic and European languages other than English are almost absent in the public space of Yemen. Yemeni Arabic featured only on 3 out of 755 signs and all of them are bottom-up ones.

In the remaining part of this section, the four strategies of multilingual writing will be elaborated individually with appropriate illustrations from the collected data. This will be followed by manipulating some of the LL themes peculiar to the public space of Yemen.

\subsection{Duplicating multilingual writing}

This type of multilingual writing represents the highest frequency. Reh (2004) defines duplicating multilingualism as "those practices in which exactly the same text is presented in more than one language. This practice acknowledges the existence of societal multilingualism, that is, the existence of more than one language in the target community, and may be a reaction to technical as well as to affective aspects of communication" (Reh, 2004: 8). Duplicating multilingual writing corresponds to what Backhaus (2007) terms as 'homophonic signs', which show complete translation or transliteration of two different languages. This means that all codes are exact translations.

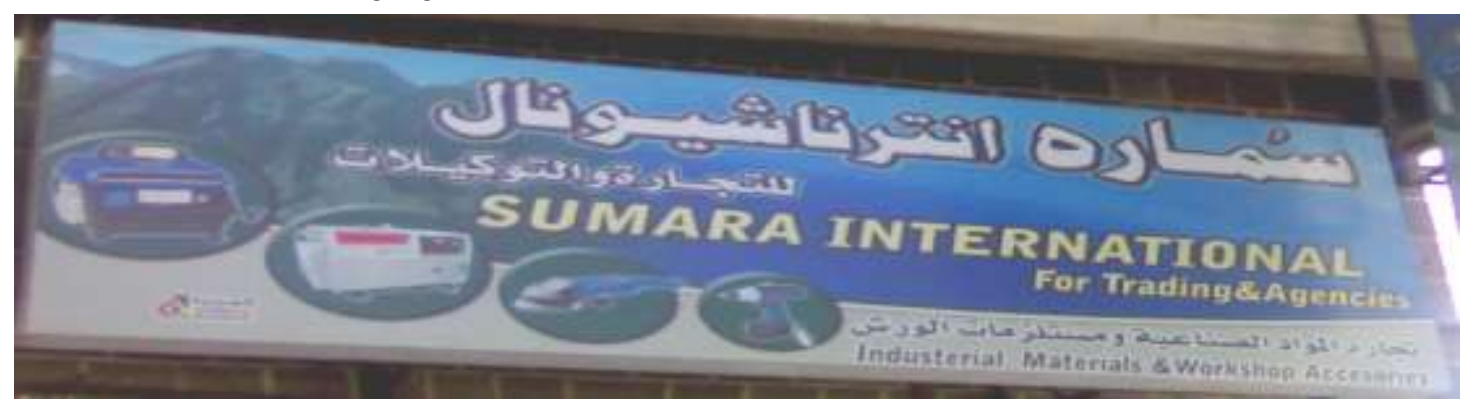

Figure 1. Duplicating multilingual writing: A commercial enterprise (bottom-up)

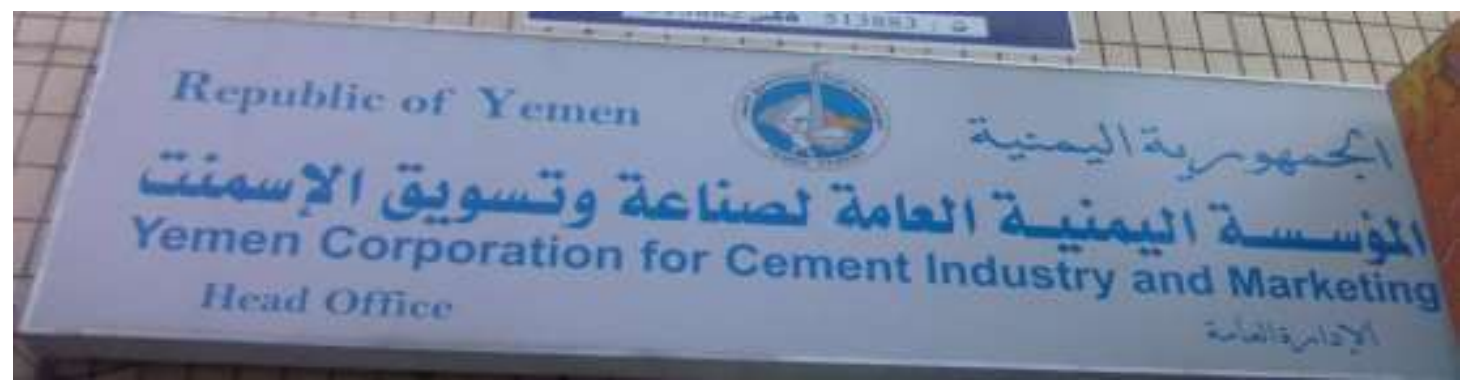

Figure 2. Duplicating multilingual writing: A government corporation (top-down)

The two signs in Figures 1 and 2 are illustrations of duplicating practices. The sign text in Figure 1, for example, includes two pieces of information: the shop name "Sumara International for trading \& Agencies" in yellow which is the exact translation of the Arabic name on the top in white and some details at the bottom about the goods provided by this commercial enterprise. The size of the two codes for each part are also similar. The second typical example in Figure 2 is a top-down sign which refers to a government cement establishment where all information, which is in Arabic, is rendered into English. This sign is multifunctional: in addition to the shop name in the middle, the text tells us that this firm is state-based one (Republic of Yemen) and gives us information about the building (head office). The English translation reinforces the image of English as the global medium to communicate with the outside world.

Reh argues that duplicating multilingual writing is used when not all members of the target group can be reached by a monolingual message or when the sender wants to reach a particular target group, such as tourists, businessmen, etc. In this sense, duplicating multilingual writing functions as an identity marker and reflects the equality of all the linguistic and cultural communities addressed. The second possibility holds true in the case of duplicating texts used in the Yemeni LL where English inscriptions are meant for tourists and foreigners. Shop owners are keen on to convey the whole message in English to the reader.

\subsection{Fragmentary multilingual writing}

This type of multilingual writing is also common in Sana'a's LL. Fragmentary multilingualism occurs when only a part of the message is translated into other languages. As Reh (2004: 10) puts it, "the term fragmentary multilingualism is used for multilingual texts in which the full information is given only in one language, but in which selected parts have been translated into an additional language or additional languages." 


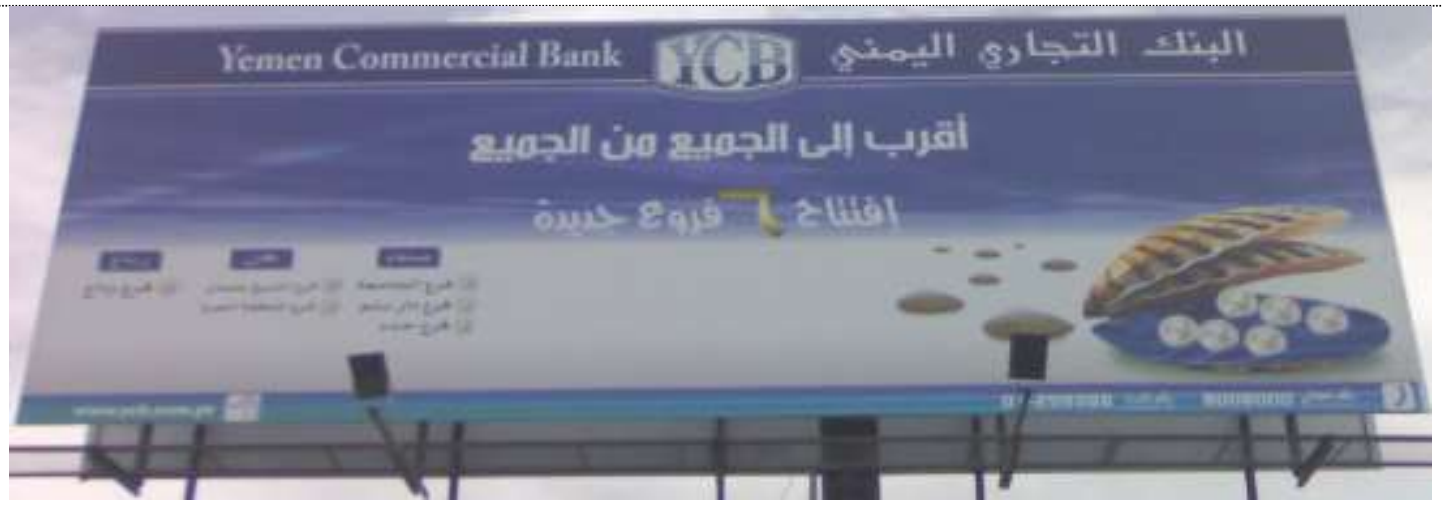

Figure 3. Fragmentary multilingual writing: A bank (top-down)

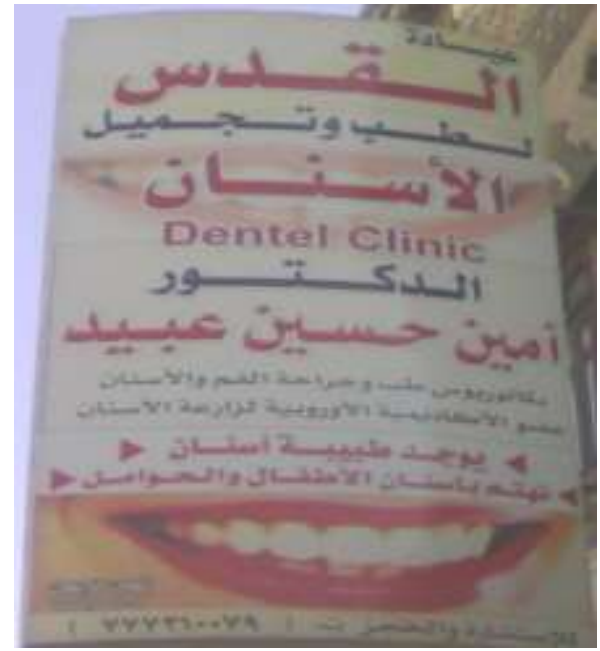

Figure 4. Fragmentary multilingual writing: A dentist's (bottom-up)

The multifunctional billboard in Figure 3 is a prime example of fragmentary multilingual writing, as put forward by Reh (2004). It is fragmentary in the sense that only one part of the inscription is converted into English, that is the name of the bank (Yemeni Commercial Bank) on the top, while other parts are in Arabic: the second line is a kind of an advertising slogan meaning "Our bank is the nearest to you", the third line informing us that the bank has opened six branches in the capital city of Sana'a, and the portion at the bottom a list of those six branches. The sign in Figure 4 is another interesting instance of fragmentary multilingualism, whose complete text is written in Arabic, while one part of the text is inscribed in English; it is obvious that Arabic is more prominent than English by virtue of complete edition. The English part "Dent(a)l Clinic" is not a translation of the clinic name as the case in Figure 3 above. It only attracts our attention to the medical service provided by the clinic which is already indicated by the image of the teeth in the bottom. So, the use of English is not meant for foreigners but as a sign of success and modernity. The sign, however, is dense with information in Arabic about the clinic name (on the top), the name of the dentist running the clinic, his qualifications (in the middle), and finally an advertisement about the availability of a female dentist for children and pregnant women.

\subsection{Overlapping multilingual writing}

Overlapping multilingualism is not very frequent. Only 17 overlapping signs are found in the corpus. Overlapping multilingual writing means that only a part of the message is repeated in another language. In all the languages also different pieces of additional information are given. In this case, the content of the texts in the different languages may simply overlap, or the content may be identical although the speech acts are not. Even though Reh (2004) draws a distinction between fragmentary and overlapping multilingual texts, Backhaus (2007) considers both types exactly the same and terms them as 'mixed signs'.

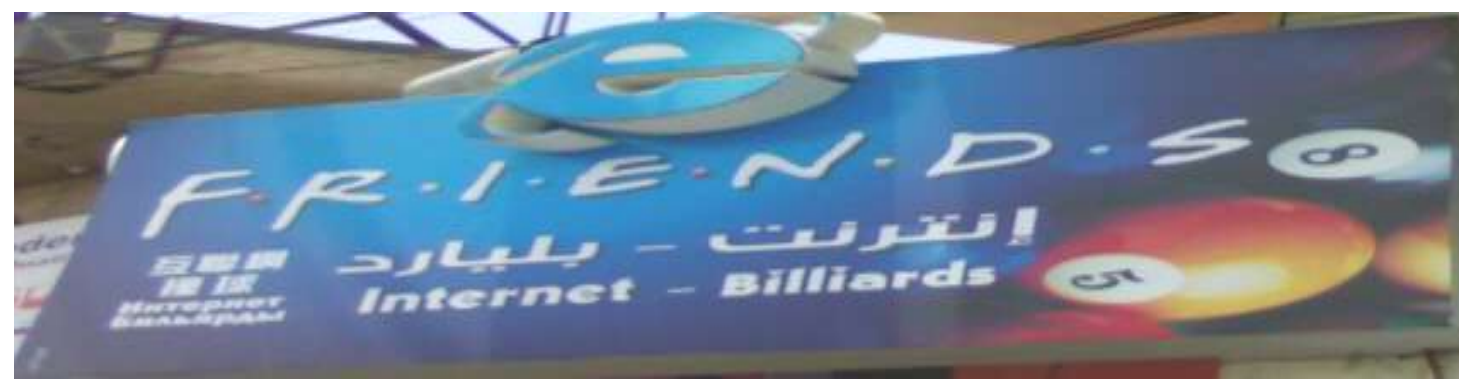

Figure 5. Overlapping multilingual writing: an internet-billiards hall (bottom-up) 


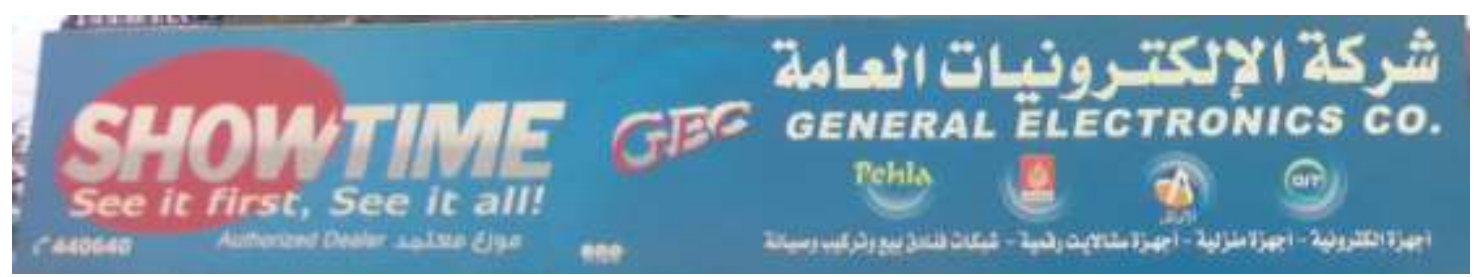

Figure 6. Overlapping multilingual writing: A commercial enterprise (bottom-up)

The inscription of the overlapping sign in Figure 5 is really unique. It has two salient features. First, it includes four languages: English, Arabic, Chinese, and Russian, respectively. Second, it is clear that the original language of the sign is English. This is indicated by introducing the name of the shop in English only (Friends) and placing it on the top. The two Arabic words in the middle which read as intarnit and bilya:rd (also bilya:rdu) are two loanwords from the English 'internet' and 'billiards'. The same two words appear in Chinese and Russian on the left side of the sign. The question which may be raised here is why English functions as the original language of the sign. The answer is two-fold. The presenting of the shop name 'friends' in this form is an exact imitation of the advertising logo for a long-running US TV serial. This marketing strategy of associating a product or a service with a foreign culture is called 'country of origin effect', a tactic that is used to imbue the marketed product or service with connotations of confidence and desirability (Buckingham and Al-Athwary 2016). The other possible explanation is related to the location of this sign. This sign is located in Haddah Street, an area which is considered to be the affluent and upper-class zone of the city and a place where many multinational companies and a number of foreign embassies are located. So, the motive behind the use of English as the original language of the sign here is not only the desire to be more prestigious, and fashionable but also to be directed at foreigners. The overlapping practice in Figure 6 lies in the insertion of the slogan "SHOWTIME ... See it first, See it all". Appearing exclusively in English, the slogan functions as a sign of glocalisation where the international brand name "SHOWTIME" is combined with the local slogan mentioned above. The other three portions of the sign are in Arabic with two of them are converted into English.

\subsection{Complementary multilingual writing}

Complementary multilingualism occurs when the different parts of the message are in different languages. In such a case, one has to be familiar with all the used languages in order to understand the sign. The difference between overlapping and complementary multilingualism is that there is some repetition in overlapping multilingualism and none in complementary (Reh 2004).

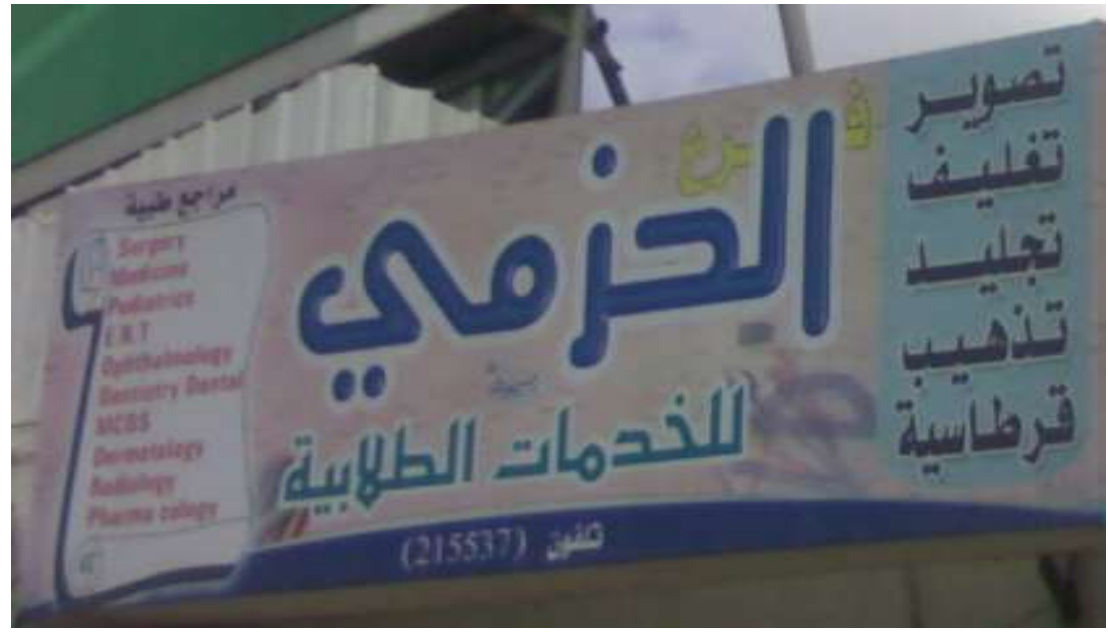

Figure 7. Complementary multilingual writing: A stationary and book shop (bottom-up)

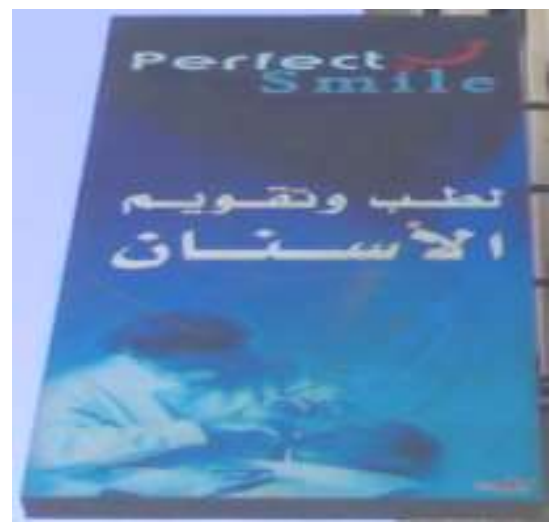

Figure 8. Complementary multilingual writing: A dentist's (bottom-up) 
The multifunctional sign in Figure 7 is a typical example of complementary multilingualism. It employs English and Arabic for a set of advertising purposes. The sign involves three portions. In the middle appears the stationary name in Arabic only which means 'The Branch of Al-Hazmi Stationary'. The portion on the right side is also in Arabic only and refers to some details about the services provided by this stationary such as photocopying, book binding, etc. Now we come to the inscription on the left side of the sign which is exclusively in English (in red). It opens with the Arabic heading mara:je tibbiyah meaning 'books of medicine'. It lists a number of medical branches/ areas for which references are available in the store. In fact, this stationary is located just in front of the main gate of Sana'a University campus. This part of the sign, therefore, is directed to the students of Medicine College who are usually bilingual in English and can figure out the whole message of the sign. The sign in Figure 8 is also another illustration of complementary multilingual writing. It is complementary in that it shows two different pieces of information in two different codes, that is English and Arabic. The dentist's name is solely in English (Perfect Smile) on the top while the inscriptions in Arabic advertise the service which reads litib wa taqwi:m al-?asna:n meaning, 'for dentistry and orthodontics'. Unless the reader is bilingual in both English and Arabic, he/ she will not be able to fully receive the message of the sign. The two semiotic images of the dentist and patient in the bottom and the smiling lips on the top help decode the two different codes of the text.

\section{Writing system mimicry}

Writing system mimicry (WSM), as introduced by Sutherland (2015), refers to the intention to create a font which is graphically similar to a different writing system, while still retaining the underlying shape and structure of the base writing system. Typographers refer to this phenomenon as 'mimicry' or 'simulation' typefaces. This sort of semiotic strategy is quite common in branding or advertising contexts. In other words, the choice to mimic another writing system may be conducted to serve some social or commercial purposes that can be interpreted sociolinguistically; WSM is employed purposefully, either for advertising or identity purposes, or both, and the identity can be ethnic or social.

The analysis of the dada reveals that there are sixteen multilingual signs which carry the feature of WSM. All of them are bottom-up signs. This confirms Sutherland's (2015: 161) claim that "as it is not apparent on any government signs, rather only on private businesses, and appears to have identity and solidarity functions, it seems to form part of the bottom-up and non-official signs." The shop owners usually resort to this semiotic strategy mainly to promote and advertise their commodities and services. The striking example in Figure 9 is really typical and conforms with Sutherland's definition of WSM stated in the theoretical background above.

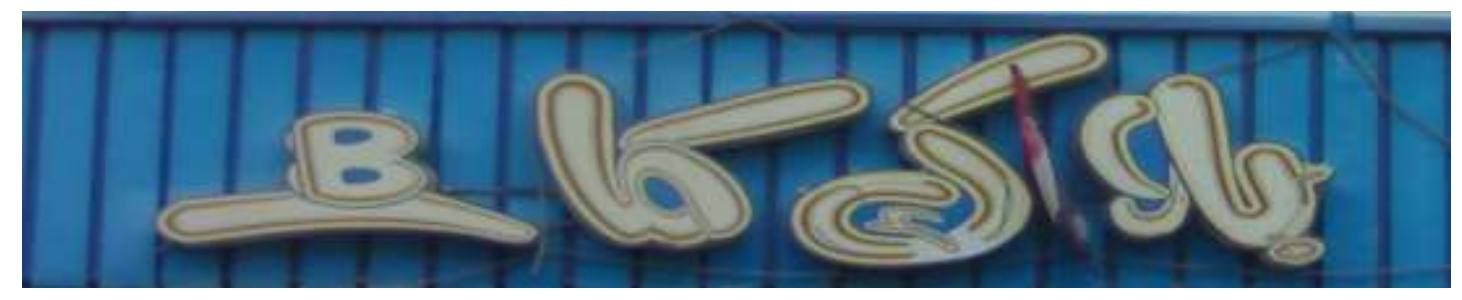

Figure 9. Two scripts in one: Black cat

To promote the business of the commercial enterprise in Figure 9, the shop name, which is originally a brand name, 'black cat', is designed uniquely and most probably the only one of its type in the whole corpus. The writer of this sign exploited the flexibility of Arabic calligraphy/ graphemes in such a way that the shop name can be read from left to right as "Black cat" and from right to left as "بلاك كات".

The mimicry process in Figure 10, however, is totally different. Here, what is mimicked is not the script of another language. Rather, the shop's name reading algharasi 'a proper family name' in the, is a mimicry of the logo of the Qatari-based Al-Jazeera television channel. The purpose, here, is also obvious; the recognizable and well-known logo of the Al-Jazeera is used as an advertising and attracting clue to promote some kind of goods.

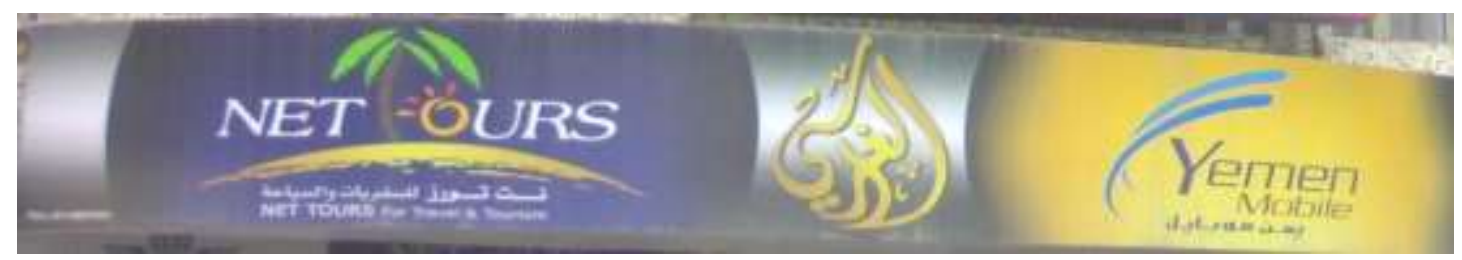

Figure 10. The mimicry of Al-Jazeera's logo 


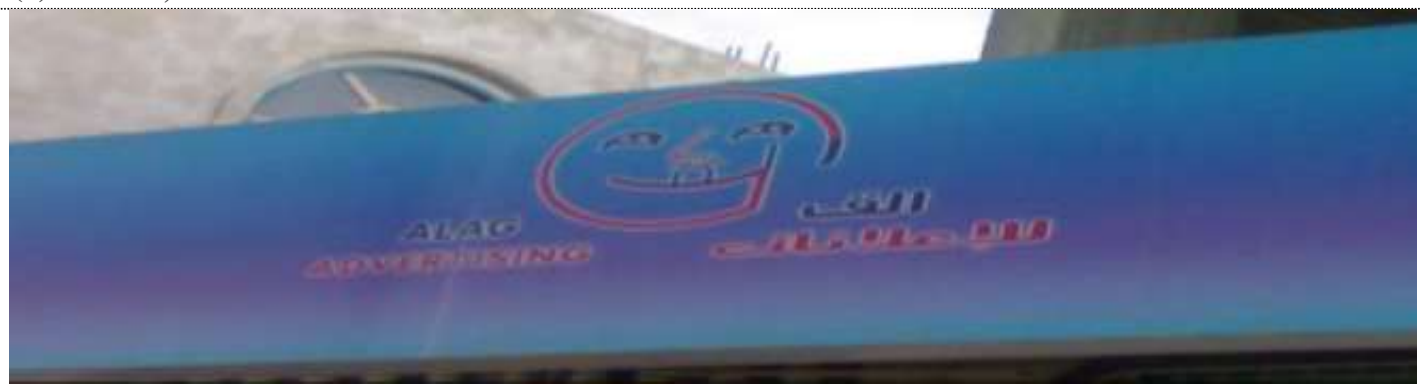

Figure 11. Face-shape mimicry: ALAG

The duplicating multilingual sign in Figure 11 is also very interesting. Like the mimicry process in Figure 10, WSM has nothing to do with mimicking the graphemes of another language. The RA shop name ALAG meaning 'glow/ brightness' is creatively depicted in the shape of a smiling face: the two As are the eyes, $\mathrm{L}$ is the nose and $\mathrm{G}$ represent the face itself. The commercial enterprise being advertised is an advertising office. The owners themselves are the designers of the sign. They create it professionally resulting in a very catchy logo for the enterprise.

\section{Discussion}

Reh's (2004) four main strategies of multilingual writing presented above determine the degree of translation and transliteration on multilingual signs. The basic difference between duplicating, fragmentary and overlapping practices, on one hand, and complementary signs, on the other, is that the latter type requires a multilingual reader if it is to be fully understood, whereas the former three types do not. The multilingual practices in Figures 7 and 8 above, for example, are not very frequent in the public space of Yemen because they target only particular group of readers, namely those who are bilingual in English. In other words, to comprehend the whole message, it is required for the reader to be competent in all languages involved. Such practices show no mutual translation for two or more languages.

Even though there are similarities observed on top-down and bottom-up signs regarding fragmentary and duplicating multilingual writing, the major differences lie in overlapping and complementary multilingualism, which reflects a nongovernmental tolerance of a wide range of languages and language varieties not visually permitted on governmentrelated signs. Consequently, these two strategies are totally absent in top-down signage process. The presence of English on both bottom-up and top-down signs reflects conscious language decisions on the part of the state and the local community to support the presence of English in the LL.

English and Arabic and some of their varieties such as Romanized Arabic (RA), Arabicized English (AE), are used in the multilingual space of Yemen. Both RA and AE are considered language varieties. RA refers to Arabic text written in Roman script, whilst AE involves English words inscribed in Arabic script. They are observed in the LL of Yemen. According to Alomoush (2016:165), the use of RA and AE on signs helps in (a) advocating linguistic tolerance and glocal identity, (b) promoting local names and cultural references and (c) creating new functions, including lexical needs and euphemisms. AE is used abundantly in Sana'a's LL, especially in duplicating and fragmentary signs. The use of $\mathrm{AE}$ is meant to assert the importance of modernity, good quality, and reconciliation with the local community via using AE in Arabic letters. The high presence of written English on other commercial signs such as business name signs is due to the mutual relation between English and success in marketing. The widespread of English in public life has made it possible that English and $\mathrm{AE}$ are used as popular mediums for expressing fondness in Western culture, especially among affluent classes.

The scarce use of Yemeni Arabic, as mentioned above, may lead us to the notion of Arab nationalism. Yemeni Arabic is never used in the top-down signs, and only 3 out of 755 signs are featured for Yemeni Arabic in bottom-up signage (cf. Alomoush, 2015). Standard Arabic (SA) appears on almost all of both top-down and bottom-up signs. It is an effect and indicative of linguistic nationalism which refers to the communicative and symbolic functions of SA in articulating national identity in the LL. In symbolic terms, "SA is the language of power and control, as opposed to the language of intimacy and domesticity of dialects, and it impinges in multifarious and sometimes subliminal ways on the daily life of Arabs of all generations, backgrounds, and educational levels" (Holes, 2004: 9). There are many regional dialects of Yemeni Arabic being used in the capital city of Sana'a. However, none of these is used in signage system due to the fact that they are predominantly spoken varieties of Arabic, used extensively in conversations and daily life activities. All Arabic inscriptions are written in SA with some exceptions in very few signs (see Figure (8a) below where the phrase muba:yl 3ala tu:l, meaning 'mobile phones forever' is in Yemeni Arabic). Although it is beyond our concern, the analysis of monolingual signs in Arabic which represents the other part of the larger corpus, surprisingly reveals that only 9 signs out of about 800 featured for Yemeni Arabic. In addition, some foreign languages like French, Chinese and Russian are used but in a very limited scope.

There is also the phenomenon of linguistic glocalisation which has emerged in multilingual writing practices. Glocalization is basically the adaptation of international products around the particularities of a local culture in which they are sold. The process allows integration of local markets into world markets. The process of glocalisation in the international arena leads to new expressions of cultural mix in music, food and clothing, but also in languages (Groter 2006). Linguistic glocalisation in our context refers to the use of international elements together with local ones as in sign Figure 6 above where the trade mark 'SHOWTIME' is mixed with the local slogan 'see it first, See it all'. Another striking example of this phenomenon is the overlapping sign in Figure 12 below. The expression YEMENTON on the left side, which also appears in AE on the top, is an imitation of the well-known tea brand name 'Lipton'. The insertion 
of 'Yemen' in the name of this international trademark adds some kind of advertising power to the slogans in Arabic and English.

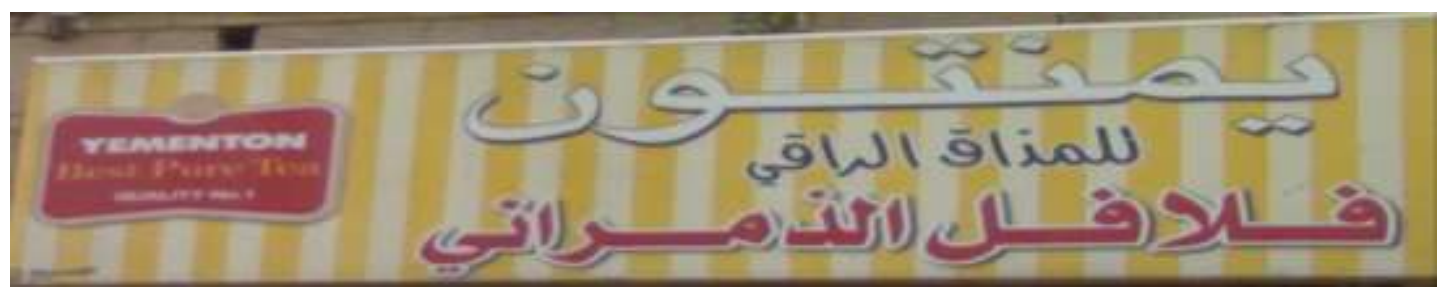

Figure 12. Glocalisation (mixed slogans)

The other phase of glocalisation is the mixing of Arabic and English elements on signs. Two mixed codes are commonly used in the LL of Yemen: RA and AE. The fragmentary sign in Figure 13 and the complementary sign in Figure 14 are good instances of this phase. In the former, "Mobile Ala Tooool" is the RA or transliteration of the Arabic shop name on the top, meaning 'cell phones forever', and the shop name in AE in the latter is the transliteration of the English phrases "Sky Net .... World Wide Express".

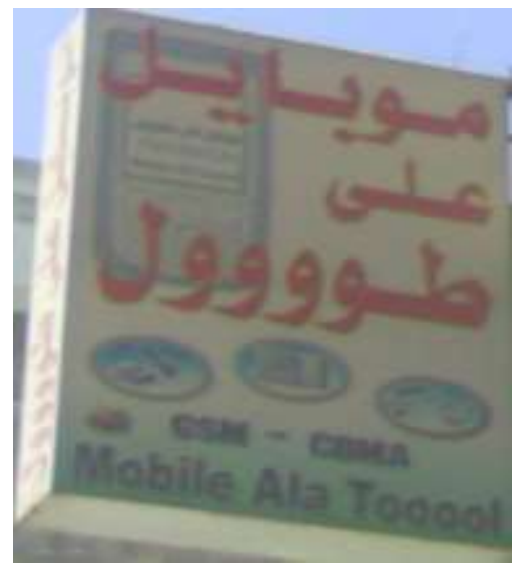

Figure 13. Glocalisation, Mixed codes: RA and EA (a mobile store)

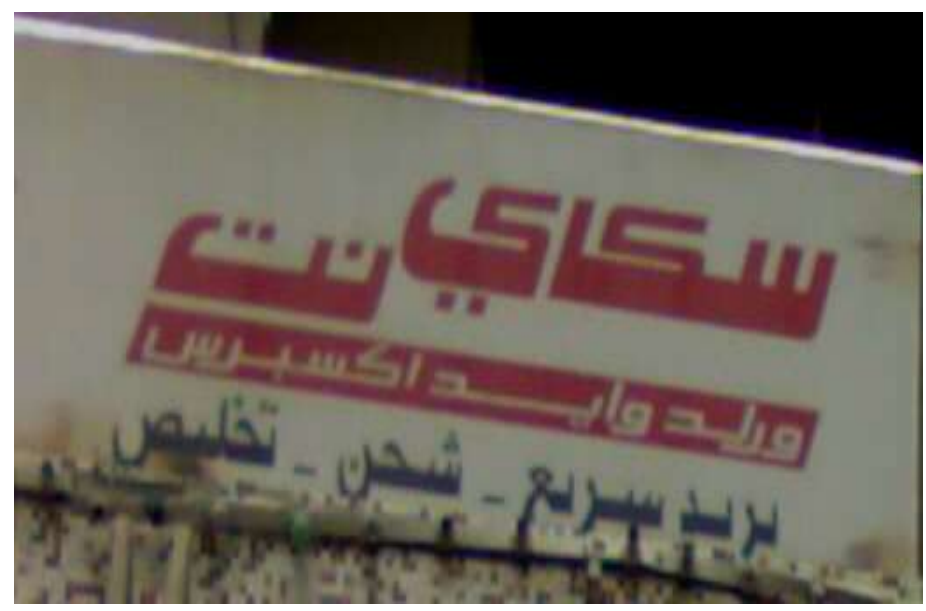

Figure 14. Glocalisation, Mixed codes: RA and EA (a cargo office)

This mixed use of RA and AE, as mentioned earlier in this section, helps shop owners to advocate glocal identity and promote local names and culture reference. Glocalisation, therefore, has become very common practice; in order to succeed globally, the concerned institutions and commercial enterprises should think globally and act locally, including the biggest multinationals.

Many of the fragmentary signs reflect the shop owner's tendency of having only one word in English while the rest of the content is in Arabic. This word specifically refers to the commodities sold or services provided by a given commercial enterprise. This practice is very frequent on the shop signs of public telephone booths, pharmacies, dental clinics, and honey selling stores where words like "telecom.", "pharmacy", "dental (clinic)" and "honey" are the only English inscriptions found, respectively (see, for example, Figure 4 above). Again such practices of multilingual writing support the view that English is used here to satisfy the desire of being modern, prestigious and successful and never meant to be directed to tourists, foreigners, etc.

In many cases also quite large spaces of the multilingual signs are devoted to advertising brand names to the detriment of multilingual writing inscriptions. The overlapping sing in Figure 6 and the fragmentary sign in Figure 10 above are good examples of this practice. In the former, the trademark SHOWTIME occupies nearly half of the sign space, and in 
the latter the brand name of the telecommunication company "Yemen Mobile" on the right side and the trademark of NET TOURS on the left side, both occupy more than two-thirds of the sign space. This advertising practice is two folds; it can be explained by the fact that the shop owners are paid for doing so or it is an advertising strategy used by the concerned companies who pay the expenses of the sign to promote their goods and services. Brand name promotion is an additional function which makes signs to be classified as multifunctional signs.

The presence of instances of WSM in section 7 above, though not very frequent, indicates that WSM has become a distinctive feature of the multilingual LL. Examples presented in Figures 4-6 above reveal that while the mimicry process in Figure 9 is in line with Sutherland's (2015) definition of WSM, Figures 10 and 11 are not. The elements being mimicked don't refer to language scripts but rather to some social and creative simulations.

Moreover, all instances of WSM found in the LL of Yemen are employed for the purpose of promotion and advertising and have nothing to do with the function of ethnic identity as may be the case in other LLs like that of London investigated by Sutherland (2015) where Arabic, Bangladeshi, etc. minorities do exist. The low frequency of WSM in Sana'a's LL, therefore, supports Sutherland's (2015) view that WSM is usually used to express the identity of ethnolinguistic minorities like those instances found in the LL of London.

\section{Conclusion}

A close examination of public space of Yemen has revealed that the LL in Yemen is much more multilingual than one may expect. The paradox is that while speech community in Yemen is monolingual in Arabic, the LL there is characterized by multilingualism. In applying Reh's (2004) reader-oriented typological model of multilingualism, the analysis shows that the four strategies of multilingual writing are attested with varying degrees of manifestation. Duplicating and fragmentary multilingualism record higher frequency than overlapping and complementary multilingual writing in both bottom-up and top-down signs. Overlapping and complementary multilingual texts presuppose multilingual readers since knowledge of all the languages involved is necessary to understand the whole message. This accounts for the paucity of occurrence of these two strategies which takes place due to the fact that the overwhelming majority of Yemenis are monolingual in Arabic. The non-existence of overlapping and complementary multilingual inscriptions in top-down signs reflects the policy on the part of local authority which doesn't tolerate the use of a wide range of languages and language varieties not visually permitted on government-related signs. Themes like the use AE, glocalization, and WSM are found to be salient features of the public space of Yemen. The use of AE is meant to assert the importance of modernity, good quality, and reconciliation with the local community. The widespread of English in public life has made it possible that English and AE are used as popular mediums for expressing fondness in Western culture, especially among affluent classes. Linguistic glocalisation in our context refers to the use of international elements together with local ones serving the purpose of adding some kind of advertising power to the slogans in Arabic and English. Finally, WSM is found to perform peculiar functions in Sana'a LL; it is only used for advertising and promotional purposes rather than expressing the identity of ethnolinguistic minorities.

The LL of Yemen, especially in Aden and Hodeida, still needs to be investigated from a diachronic or, what is called by Backhaus (2005), layering perspective; indoor (universities, hospitals, malls, store departments, etc.) and movable signs, posters, and advertisements represent a fertile area of study within the framework of LL in Yemen (see AlAthwary, 2012). A comparative study of multilingual practices and language policy in Yemen and other Arab countries like Saudi Arabia, and Jordan, for example, is required to be conducted. Writing system mimicry has been shown to be a distinctive feature of the linguistic landscape. It is a rich source of sociolinguistic research that has not been examined enough. This study leaves the door open for pursuing an investigation in this area of research.

\section{References}

Akindele, D. (2011). Linguistic Landscapes as Public Communication: A Study of Public Signage in Gaborone Botswana. International Journal of Linguistics, 3(1), 1-11.

Al-Athwary, A. (2012). Exploring the Structure and Functions of Sana'a's Linguistic Landscape. Journal of Social Studies, 34, 9- 45.

Al-Athwary, A. (2014). Translating Shop Signs into English in Sana'a's Streets: A Linguistic Analysis. International Journal of Humanities and Social Science, 4 (12), 140- 156.

Alomoush, O. (2015). Multilingualism in the linguistic landscape of urban Jordan. PhD thesis, University of Liverpool, Liverpool, UK.

Anuarudin, A., Heng, C., and Abdullah, A. (2013). Exploring Multilingual Practices in Billboard Advertisements in a Linguistic Landscape. Pertanika Journal of Social Sciences \& Humanities, 21 (2): 783 - 796.

Backhaus, P. (2005). Signs of multilingualism in Tokyo- a diachronic look at the linguistic landscape. Int'l J. Soc. Lang., 175/ 176, $103-121$.

Backhaus, P. (2007). Linguistic landscapes: a comparative study of urban multilingualism in Tokyo. Clevedon: Multilingual Matters.

Ben Said, S. (2010). Urban Street Signs in the Linguistic Landscape of Tunisia: Tensions in Policy, Representation, and Attitudes. Ph.D. thesis, the Pennsylvania State University, USA.

Ben-Rafael, E. (2009). A sociological approach to the study of linguistic landscapes. In: Gorter D. and Shohamy E. (eds.). Linguistic landscape: expanding the scenery. New York: Routledge, 189-205. 
Ben-Rafael, E., Shohamy, E., Amara, M., and Trumper-Hecht, N. (2006). Linguistic Landscape as Symbolic Construction of the Public Space: The Case of Israel. International Journal of Multilingualism, 3 (1), 7 - 30.

Bolton, K. (2012). World Englishes and linguistic landscapes. World Englishes, 31, (1), 30-33.

Buckingham, L. (2015). Commercial signage and the linguistic landscape of Oman. World Englishes. 34(3), 411-435.

Buckingham, L. and Al-Athwary, A. (2016). Commercial signs in Oman and Yemen: A study of street advertising in English. In Buckingham, L. (ed.). Language, Identity, and Education on the Arabian Peninsula Bilingual Policies in a Multilingual Context. Bristol: Multilingual Matters.

Cenoz, J. and Gorter, D. (2006). Linguistic landscape and minority languages. In: Gorter, D. (ed.), Linguistic landscape: A New Approach to Multilingualism. Clevedon: Multilingual Matters, 67-81.

Cenoz, J. and Gorter, D. (2008). Linguistic landscape as an additional source in second language acquisition. International Review of Applied Linguistics in Language Teaching, 46, 267- 287.

Clemente, M., Vieira, R., Martins, F., Andrade, A. (2013). Linguistic Diversity in Aveiro, Portugal: Exploring Linguistic Landscape Methodologies in the «Beira Mar» Neighborhood. Internet Latent Corpus Journal, 3 (1), 116133.

Coulmas, F. (2014). Writing systems and language contact in the Euro- and Sinocentric worlds. Applied Linguistics Review, 5(1), $1-21$.

Edelman, L. (2010). Linguistic landscapes in the Netherlands: a study of multilingualism in Amsterdam and Friesland. LOT: the Netherlands National Graduate School of Linguistics, the Netherlands. The University of Amsterdam.

Gorter, D. (2006). Further Possibilities for Linguistic Landscape Research. In Gorter, D. (ed.), Linguistic Landscape: A New Approach to Multilingualism. Clevedon: Multilingual Matters, 81- 89.

Gorter, D. (2013). Linguistic Landscapes in a Multilingual World. Annual Review of Applied Linguistics, 33, 190-212.

Haynes, P. (2012). Welsh Language Policy: A Study of the Linguistic Landscape at Cardiff University. M.A. dissertation, the University of Birmingham, Birmingham.

Holes, C. (2004). Modern Arabic Structures, Functions, and Varieties. Georgetown University Press, Washington D.C.

Landry, R. and Bourhis R. (1997). Linguistic landscape and ethnolinguistic vitality: an empirical study. Journal of Language and Social Psychology, 16(1), 23-49.

Nash, J. (2016). Is linguistic landscape necessary? [Review article of the books Ethnography, superdiversity and linguistic landscapes: chronicles of complexity, by J. Blommaert and Linguistic landscapes, multilingualism, and social change, by C. Hélot, M. Barni, R. Janssens and C. Bagna]. Landscape Research, 41(3), 380-384.

Nofal, M. and Mansour, A. (2014). The Linguistic Landscape in Jordan: Opinions and Attitudes. Global Journal of Interdisciplinary Social Studies, 3(3), 27-37.

Puzey, G. (2016). Linguistic landscapes. In C. Hough (ed.) Oxford handbook of names and naming. Oxford: Oxford University Press, 395-411.

Reh, M. (2004). Multilingual writing: A reader-oriented typology - With examples from Lira Municipality (Uganda). International Journal of the Sociology of Language, 170, 1-41.

Seargeant, P. (2013). Between Script and Language: The ambiguous ascription of 'English' in the Linguistic Landscape. In Hélot, C., Barni, M., Janssens, R., Bagna, C. (eds.), Linguistic Landscapes, Multilingualism and Social Change. Frankfurt am Main, Berlin, Bern, Bruxelles, New York, Oxford, Wien, 187-200.

Sebba, M. (2012). Multilingualism in written discourse: An approach to the analysis of multilingual texts. International Journal of Bilingualism, 17(1), 97-118.

Shohamy, E., Ben-Rafael, E. and Barni, M. (2010). Introduction. In Shohamy, E., Ben-Rafael, E. and Barni, M (eds.). Linguistic landscape in the city. Bristol: Multilingual Matters, xi- xv.

Singhasiri, W. (2013). Linguistic Landscape in the State Railway Station of Thailand: The Analysis of the Use of Language. Presented at The European Conference on Language Learning 2013, Brighton, UK, 18-19 July.

Spolsky, B. (2004). Language policy. Cambridge: Cambridge University Press.

Sutherland, P. (2015). Writing System Mimicry in the Linguistic Landscape. SOAS Working Papers in Linguistics, 17, 147-167.

Troyer, R. (2012). English in the Thai linguistic netscape. World Englishes, 31(1), 93-112.

Tufi, S. and Blackwood, R. (2010). Trademarks in the linguistic landscape: methodological and theoretical challenges in qualifying brand names in the public space. International Journal of Multilingualism, 7 (3), 197-210.

Wielfaert, T. (2009). A Linguistic Ethnographic Study of Written Multilingual Signage on a South African University Campus. M.A. dissertation, Ghent University, Ghent, Belgium. 